\title{
Factors associated with intention to undergo specific health guidance among Japanese workers using health belief model
}

\author{
Kumiko Yamamoto, Marie Mizoshita, Rie Akamatsu* \\ Graduate School of Humanities and Sciences, Ochanomizu University, Tokyo, Japan; \\ *Corresponding Author: akamatsu.rie@ocha.ac.jp
}

Received 7 January 2012; revised 18 February 2012; accepted 21 March 2012

\section{ABSTRACT}

Objectives: To examine the factors associated with the intention to undergo "specific health guidance". Methods: Self-reported questionnaire data were collected from 4861 health insurance union members of a company in Japan from August to September 2010. The information gathered included the intention to undergo "specific health guidance," the Health Belief Model (HBM) subcomponents (perceived severity, susceptibility, benefit, and barriers), other factors related to undergoing specific health guidance, sex, and age. Perceived threat and net benefit were the main HBM components. Bivariate and multivariate logistic regression analyses were used to assess the associations between intention and the main HBM components, i.e., perceived threat and net benefit. We used two models with intention as the dependent variable; Model 1 used four HBM subcomponents, and Model 2 used perceived threat and net benefit as independent variables. Results: A total of 3457 individuals answered all variables (response rate; $71.1 \%$ ). Perceived severity (OR: 1.83, 95\% Cl: 1.59 - 2.10), benefit (OR: 6.91, 95\% Cl: 5.94 - 8.04), barriers (OR: $0.11,95 \% \mathrm{Cl}: 0.09-0.13$ ), perceived threat (OR: $1.52,95 \% \mathrm{Cl}: 1.31-1.76$ ), and net benefit (OR: $11.28,95 \% \mathrm{Cl}$ : $9.60-13.25$ ) were related to intention (all $p<0.01$ ). Multivariate analysis showed that net benefit (OR: 11.23, 95\% Cl: 9.55 - 13.20) had a stronger correlation with intention than did perceived benefit (OR: 4.67, 95\% Cl: 3.95 - 5.51) and barriers (OR: $0.15,95 \% \mathrm{Cl}: 0.13-0.18$ ) (all $p<0.01$ ). Conclusions: The results suggested that the main HBM components may predict health behavior better than the subcomponents. These results may effectively promote the benefits of, and reduce barriers to, pro- grams aiming to increase participation in specific health guidance.

Keywords: Patient Participation; Health Belief Model; Net Benefit; Japanese; Health Guidance

\section{INTRODUCTION}

Among 40 - 74 year-old individuals in Japan, 50\% of males and $20 \%$ of females are strongly suspected to have either metabolic syndrome or prodromal metabolic syndrome $[1,2]$. Therefore, healthcare insurance providers have been mandated by Japanese law to provide health checkups to their insured customers between the ages of 40 and 74 since April 2008 [3]. Moreover, insurance providers must give people at risk of metabolic syndrome opportunities to undergo a health consultation, which is called "specific health guidance" [3]. People who undergo specific health guidance achieve positive health outcomes, such as weight loss and decreased hemoglobin A1c and serum triglyceride levels [4-6]. However, the specific health guidance completion rate is low $(7.7 \%$ in $2008,12.8 \%$ in 2009) $[7,8]$. Thus, it is necessary to investigate why people choose to participate in or forego specific health guidance.

Most of those targeted for specific health guidance are workers; thus, they have various reasons not to participate in health promotion programs. Studies indicated that a lack of time or being busy was the most common reason not to participate in worksite health-promotion (WHP) programs [9-14]. For example, Gucciardi and colleagues reported that people preferred to participate in one 30 - 60 min education intervention session a year [15]. Cost was also an important problem for participation in WHP programs [12,16], and inconvenient location was also identified as a barrier [9]. Thus, overcoming these obstacles is desirable.

In addition to practical barriers, a perceived lack of benefit, feeling non-susceptible to illness, and a lack of 
motivation were cited as reasons not to participate in WHP programs $[10,16]$. This suggests that people would take part in WHP programs if they felt them to be beneficial and were thus motivated to participate. Indeed, perceived benefit was shown to facilitate participation in various health-promotion programs [12,17]. Previous studies investigated these benefits, such as being healthy, feeling good, weight loss, improved appearance, and participation in fun activities [12,17,18]. Additionally, although few studies have examined the benefits and barriers that are more strongly correlated with participation in WHP programs, both benefits and barriers are correlated with intention to participate [17,19].

In the Health Belief Model (HBM), which describes why individuals engage in health behaviors using factors such as perceived susceptibility, perceived severity, perceived benefits, and perceived barriers, Janz and Becker subtracted the perceived barriers from the perceived benefit. The resulting difference score (net benefit) has a direct effect on behavior [20]. The other main component of HBM is perceived threat. This derives from two subcomponents: perceived severity and perceived susceptibility. Perceived severity is an individual's belief about the severity or seriousness of a disease. Perceived susceptibility is the perception of susceptibility to a disease. Threat perception is calculated as the product of perceived severity and perceived susceptibility, and it has a direct effect on behavior. In essence, HBM hypothesizes that if individuals regard themselves as susceptible to a condition, believe that condition has potentially serious consequences, believe that a course of action available to them would be beneficial in reducing either their susceptibility to or severity of the condition, and believe that the anticipated benefits of taking action outweigh the barriers to it, they are likely to take action that they believe will reduce their risk [21].

In HBM, the factors directly influencing behavior are perceived threat and net benefit; however, the HBM include four subcomponents: perceived severity, perceived susceptibility, perceived benefit, and perceived barriers. Therefore, most authors have used these four subcomponents in their analysis [22,23]. Perceived benefit and perceived barriers were strong predictors of behavior [22-26]. Perceived severity and perceived susceptibility were also predictors of behavior, but the correlations were weak or not significant, depending on the study [24,26,27].

Although the correlations between a behavior and the four subcomponents have been identified, the combined influence of the four subcomponents has not. Few studies have analyzed perceived threat and net benefit. One study of risky driving behaviors indicated that three of the four subcomponents, the exception being perceived barriers, failed to predict the likelihood of risky driving behavior, but perceived threat and net benefit did [28] Thus, we investigated the predictors of intention to undergo specific health guidance using the main HBM components: perceived threat and net benefit. The purpose of this study was to investigate the predictors of intention to undergo specific health guidance using these HBM components. We hypothesized that perceived threat and net benefit would be associated with intention.

\section{METHODS}

\subsection{Participants and Procedure}

Subjects were 4861 Japanese health insurance union members. From August to September, 2010, anonymous self-administered questionnaires (original version in Japanese) and envelopes for their return were enclosed with a household medicine application form. Subjects returned questionnaires with the application form to the health insurance society. Next, the applicant ID on the questionnaire was used by the health insurance society to identify the health insurance member, and the authors received the questionnaires, ID, and information on sex and age. The study received approval from the Ochanomizu University Ethics Review Committee.

\subsection{Measures}

The questionnaires included questions about future intention to undergo specific health guidance, the HBM factors (perceived severity and perceived susceptibility for lifestyle diseases, and perceived benefits of and perceived barriers to undergoing specific health guidance), and other factors (recommendation by associates, family history of lifestyle disease, and self-rated health).

\subsubsection{Intention}

The intention to undergo specific health guidance was determined using the question "Will you undergo specific health guidance if you become a subject of guidance?” Participants answered on a 5-point scale [never undergo (1) to definitely undergo (5)].

\subsubsection{HBM Subcomponents}

Perceived severity of lifestyle diseases (perceived severity) was assessed by the question, "Do you think that lifestyle disease is serious illness?” Perceived susceptibility to lifestyle diseases (perceived susceptibility) was asked using the question, "Compared to other people your age, do you think that your chance of having lifestyle disease in the future is higher?" Perceived benefit of specific health guidance (perceived benefit) was determined as follows: "Do you think that you can prevent lifestyle diseases if you undergo specific health guidance?” Perceived barriers to specific health guidance 
(perceived barriers) were identified using one item, “Do you have any reason not to undergo specific health guidance?" Participants answered each item using a 5-point scale [strongly disagree (1) to strongly agree (5)].

Additionally, we asked subjects who endorsed a perceived barrier with a score between 2 and 5 (i.e., those who perceived a barrier) to identify their reasons for not undergoing specific health guidance. Participants responded by answering "yes" or "no" to each of eight items.

\subsubsection{Other Factors Related to Undergoing Specific Health Guidance}

Recommendation by associates (recommendation) was asked thus: "Have you ever received any recommendation about modifying your lifestyle from people around you such as family, friends, and physicians?” Participants answered on a 5-point scale [strongly disagree (1) to strongly agree (5)].

Family history of lifestyle diseases (family history) was determined with one item: "Have your parents, siblings, grandparents, uncles, or aunts ever been diagnosed with lifestyle diseases?" Participants were allowed to provide multiple answers.

Self-rated health was asked using one item: "How has the condition of your health been recently?” Participants answered on a 5-point scale [extremely bad (1) to extremely good (5)].

\subsection{Statistical Analyses}

A total of 3645 individuals responded to the questionnaire (response rate, 75.0\%), and 3457 answered all variables (valid response rate, $71.1 \%$ ). Sample size for an effect size of 0.1 using an alpha of 0.01 and beta of 0.8 was calculated to be 1169 . Therefore, this study had sufficient power. Frequencies and median (interquartile range: IRQ) were determined for basic attributes, and the Mann-Whitney test and Spearman's correlation coefficient were conducted to assess the associations between intention and basic attributes. New variables termed "perceived threat" and "net benefit” were generated. Perceived threat was calculated by multiplying the perceived severity score by the perceived susceptibility score, and net benefit was calculated by subtracting the perceived barriers score from the perceived benefit score. Then, each Likert-scale item was transformed to binary variables by dichotomizing the scores at the median score (See Tables 1 and 2).

Bivariate and multivariate logistic regression analyses were conducted to evaluate the associations between intention and the four HBM subcomponents, perceived threat, and net benefit. Bivariate and multivariate logistic regression analyses were conducted with intention as the dependent variable and the HBM factors as independent variables. In the multivariate analysis, the independent variables were the four HBM subcomponents from Model

Table 1. Frequency distribution and bivariate/multivariate logistic regression analyses of perceived severity, susceptibility, benefit, and barriers as predictors of intention $(n=3457)$.

\begin{tabular}{|c|c|c|c|c|c|}
\hline \multirow{2}{*}{ Independent variables } & \multirow{2}{*}{ Total $n(\%)$} & \multicolumn{2}{|c|}{ Intention } & \multirow{2}{*}{$\begin{array}{c}\text { Bivariate }^{\mathrm{a}} \\
\text { OR }(95 \% \mathrm{CI})\end{array}$} & \multirow{2}{*}{$\begin{array}{l}\text { Multivariate }^{\mathrm{b}} \\
\text { OR (95\% CI) }\end{array}$} \\
\hline & & Low $(1-3) n(\%)$ & High (4 - 5) $n(\%)$ & & \\
\hline \multicolumn{6}{|l|}{ Perceived severity } \\
\hline Low $(1-4)$ & 1507 (43.6) & $956(50.6)$ & $551(35.1)$ & Ref & Ref \\
\hline High (5) & $1950(56.4)$ & $932(49.4)$ & $1018(64.9)$ & $1.83(1.59-2.10)^{* *}$ & $1.52(1.29-1.80)^{* *}$ \\
\hline \multicolumn{6}{|l|}{ Perceived susceptibility } \\
\hline Low $(1-3)$ & 2066 (32.5) & $1116(59.1)$ & $950(60.5)$ & Ref & Ref \\
\hline High (4 - 5) & $1391(40.2)$ & $772(40.9)$ & 619 (39.5) & $1.03(0.88-1.20)$ & $1.08(0.90-1.30)$ \\
\hline \multicolumn{6}{|l|}{ Perceived benefits } \\
\hline Low $(1-3)$ & $1912(55.3)$ & 1427 (75.6) & 485 (30.9) & Ref & Ref \\
\hline High (4 - 5) & 1545 (44.7) & $461(24.4)$ & $1084(69.1)$ & $6.91(5.94-8.04)^{* *}$ & $4.67(3.95-5.51)^{* *}$ \\
\hline \multicolumn{6}{|l|}{ Perceived barriers } \\
\hline Low $(1-2)$ & $1949(56.4)$ & 648 (34.3) & 1301 (82.9) & Ref & Ref \\
\hline High (3 - 5) & 1508 (43.6) & $1240(65.7)$ & $268(17.1)$ & $0.11(0.09-0.13)^{* *}$ & $0.15(0.13-0.18)^{* *}$ \\
\hline
\end{tabular}

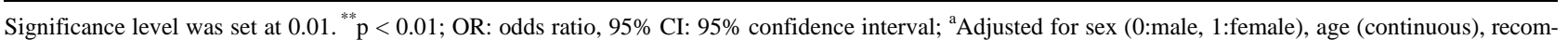
mendation [0: low (1 - 2), 1: high (3 - 5)], family history (0: none, 1: one and more), self-related health [0: low (1 - 3), 1: high (4 - 5)]; ${ }^{\mathrm{b}}$ Adjusted for covariates and other independent variables in the bivariate analysis. 
Table 2. Frequency distribution and bivariate/multivariate logistic regression analysis of perceived threat and net benefit as predictors of intention $(n=3457)$.

\begin{tabular}{|c|c|c|c|c|c|}
\hline \multirow{2}{*}{ Independent variables } & \multirow{2}{*}{ Total $n(\%)$} & \multicolumn{2}{|c|}{ Intention } & \multirow{2}{*}{$\begin{array}{c}\text { Bivariate }^{\mathrm{a}} \\
\text { OR (95\% CI) }\end{array}$} & \multirow{2}{*}{$\begin{array}{l}\text { Multivariate }^{\mathrm{b}} \\
\text { OR }(95 \% \text { CI })\end{array}$} \\
\hline & & low $(1-3) n(\%)$ & high (4 - 5) $n(\%)$ & & \\
\hline \multicolumn{6}{|l|}{ Perceived threat } \\
\hline Low (1 - 12) & 1448 (41.9) & $854(45.2)$ & 594 (37.9) & Ref & Ref \\
\hline High (15 - 25) & 2009 (58.1) & $1034(54.8)$ & 975 (62.1) & $1.52(1.31-1.76)^{* *}$ & $1.48(1.25-1.76)^{* *}$ \\
\hline \multicolumn{6}{|l|}{ Net benefit } \\
\hline Low $(-4-+1)$ & $1976(57.2)$ & $1538(81.5)$ & 438 (27.9) & Ref & Ref \\
\hline High $(+2-+4)$ & $1481(42.8)$ & 350 (18.5) & $1131(72.1)$ & $11.28(9.60-13.25)^{* *}$ & $11.23(9.55-13.20)^{* *}$ \\
\hline
\end{tabular}

Significance level was set at $0.01 .{ }^{* *} \mathrm{p}<0.01$; OR: odds ratio, 95\% CI: 95\% confidence interval; ${ }^{\mathrm{a}}$ Adjusted for sex (0: male, 1 : female), age (continuous), recommendation [0: low (1 - 2), 1: high (3 - 5)], family history (0: none, 1: one and more), self-related health [0: low (1 - 3), 1: high (4 - 5)]; ${ }^{\mathrm{b}}$ Adjusted for covariates and other independent variables in the bivariate analysis.

1 and perceived threat and net benefit from Model 2. In the logistic regression analysis, we entered sex, age, recommendation, family history, and self-rated health as control variables.

Finally, the frequency of reasons not to undergo specific health guidance were summarized.

Statistical significance was considered at $\mathrm{p}<0.01$, and all tests were two-sided. Statistical analyses were conducted using SPSS, version 19 (IBM Japan, Ltd., Tokyo, Japan).

\section{RESULTS}

\subsection{Descriptive Information}

The majority of subjects were female (57.1\%). The age of subjects ranged from 22 to 68 years, with a median (IQR) of 39.0 (31.0 - 47.0).

The median (IQR) intention score for males was 3.0 (3.0 - 4.0), and that for females was $3.0(3.0$ - 4.0). There was no significant correlation between intention and sex $(U=1,456.9, \mathrm{p}=0.80)$ or between intention and age (Spearman's $r=-0.026, \mathrm{p}=0.13$ ).

\subsection{Bivariate Correlation between Intention and HBM Components}

A bivariate logistic regression analysis revealed a bivariate correlation between HBM components and intention (Tables 1 and 2). Perceived severity (OR: 1.83, 95\% CI: 1.59 - 2.10), perceived benefit (OR: 6.91, 95\% CI: 5.94 - 8.04), perceived barriers (OR: $0.11,95 \% \mathrm{CI}$ : 0.09 - 0.13), perceived threat (OR: 1.52, 95\% CI: 1.31 1.76), and net benefit (OR: 11.28, 95\% CI: 9.60 - 13.25) were correlated with intention, and all associations were statistically significant (all $\mathrm{p}<0.01$ ). With the exception of perceived barriers, the direction of all correlations was positive.

\subsection{Multivariate Correlation between Intention and HBM Components}

A multivariate logistic regression analysis was performed to examine the correlation of perceived severity, perceived susceptibility, perceived benefit, and perceived barriers with intention after adjusting for each factor. As shown in Table 1, perceived severity (OR: 1.52, 95\% CI: 1.29 - 1.80) and perceived benefit (OR: 4.67, 95\% CI: 3.95 - 5.51) showed positive correlations with intention, and perceived barriers showed a negative correlation (OR: 0.15, 95\% CI: 0.13 - 0.18); this model had a discrimination rate of $75.6 \%$.

According to the HBM, perceived threat and net benefit directly influence the likelihood of committing an action. A multivariate regression analysis was also performed to examine the correlation of these two HBM variables with intention. Both were statistically significant predictors of intention; this model had a discrimination rate of $77.2 \%$. Although net benefit had a stronger correlation with intention (OR: 1.48, 95\% CI: 1.25 1.76) than did perceived benefit and perceived barriers, perceived threat had a weaker correlation (OR: 11.23, 95\% CI: 9.55 - 13.20) than did perceived severity (Table 2).

\subsection{Barriers to Undergoing Specific Health Guidance}

Table 3 summarizes the barriers to undergoing specific health guidance as reported by study participants. Of the total, 2473 subjects rated perceived barriers as 2 $5(2473 / 3457=71.5 \%)$, and 1981 provided reasons not to undergo specific health guidance $(1981 / 2473=$ 80.1\%). "Can't take the time" and "Don't know contents" were the most frequently reported reasons not to undergo "specific health guidance". Think that specific 
Table 3. Reasons not to undergo specific health guidance ( $n=$ 1981).

\begin{tabular}{|c|c|c|}
\hline Barriers & $n$ & $\%$ \\
\hline Can't take time & 1207 & 60.9 \\
\hline Don't know contents & 1014 & 51.2 \\
\hline Distant location & 291 & 14.7 \\
\hline $\begin{array}{l}\text { Don't want colleagues to notice that I am } \\
\text { undergoing specific health guidance }\end{array}$ & 229 & 11.6 \\
\hline $\begin{array}{l}\text { Able to improve my lifestyle by myself without } \\
\text { specific health guidance }\end{array}$ & 146 & 7.4 \\
\hline Seeing a doctor regularly & 129 & 6.5 \\
\hline $\begin{array}{l}\text { Think that specific health guidance have no } \\
\text { effect }\end{array}$ & 125 & 6.3 \\
\hline Don't want to change current lifestyle & 115 & 5.8 \\
\hline
\end{tabular}

health guidance has no effect and “Don't want change my current lifestyle" were the least common reasons.

\section{DISCUSSION}

We investigated predictors of the intention to undergo specific health guidance using the HBM components. Perceived threat, net benefit, perceived severity, perceived benefit, and perceived barriers, were associated with intention, and net benefit was a stronger predictor than perceived benefit and perceived barriers.

Our data indicate that net benefit was a stronger predictor of intention than were perceived benefit and perceived barriers. Other studies have investigated perceived benefit/barriers to participation in health-promotion programs $[9-12,14,16,18,19]$. Some of these analyzed the correlation between benefit/barriers and participation without integrating the two components [12, 17-19]. Thus, there is little information regarding net benefit. However, some studies of health behavior have examined decisional balance [21,22]. These suggested that individuals in the action/maintenance stage perceived more pros (benefits) than cons (barriers), and those in the pre-contemplation stage perceived more cons than pros [21,22]. In this study, people who perceived more benefit than barriers had a greater intention to participate, so our finding is consistent these studies [21,22]. This finding suggests the importance of the combined influence of perceived benefit and perceived barriers, demonstrating that both elevating perceived benefit and reducing perceived barriers are important. Such a change may increase the difference between perceived benefit and perceived barriers and thereby improve participation rates.

We also investigated the reasons that participants did not undergo specific health guidance. The most common answers were “can't take time” and “don't know contents." Many previous studies have reported that time was the most important barrier to participation in WHP programs [9-14]. However, Middlestadt and colleagues reported that individuals with high intention to participate in a worksite wellness program regarded programs that take place outside of work hours or during work hours and at a convenient location as facilitators of participation [12]. Thus, time is a particularly important factor because it can represent both a benefit of and a barrier to WHP programs. Few studies have examined the "don't know contents" rationale, but Fletcher and colleagues reported that a lack of instructions was one of the barriers to participation in a physical activity program [18]. Therefore, health insurance providers should explain the contents of specific health guidance to patients. These findings will be useful for developing intervention programs to improve the rate of specific health guidance participation.

Perceived threat was a significant predictor of intention, but was weaker than net benefit. This is thought to be due to the fact that perceived susceptibility was not significantly correlated with intention. Some previous studies reported that perceived susceptibility and perceived severity were significant predictors of intention/ behavior, whereas others have not found them to be predictors [22-28]. Therefore, whether perceived threat, perceived severity, and perceived susceptibility correlate with the likelihood of undergoing specific health guidance should be investigated in future research.

The present study has some limitations. First, intention was used, and whether those intentions translate into actually undergoing specific health guidance is uncertain. Second, a self-reported questionnaire was used, and the reliability of responses should be examined. Third, confounders related to the intention to undergo specific health guidance such as lifestyle, health status, working time, and support by management were not investigated and should be a focus of future research.

The strength of this study lies in the fact that we analyzed not only the four subcomponents of the HBM, but also perceived threat and net benefit; these latter two factors explained $39.1 \%$ of the variation. Therefore, the HBM can sufficiently predict intention to undergo specific health guidance.

\section{CONCLUSIONS}

The present study explored the predictors of intention of Japanese workers to undergo specific health guidance using the Health Belief Model. The model using four subcomponents (Model 1) and that using the two main components (perceived threat and net benefit; Model 2) both predicted intention; however, net benefit was a 
stronger predictor of intention than were perceived benefit or perceived barriers. Therefore, our data suggest that net benefit should be used in analyses based on the HBM, and it is important to both increase benefit and decrease barriers to improve participation in health-promotion programs.

\section{REFERENCES}

[1] Ministry of Health and Welfare (2006) National Health and Nutrition Survey. http:/www.mhlw.go.jp/houdou/2008/04/dl/h0430-2c.pdf

[2] Office for Life-Style Related Diseases Control Health Service Bureau Ministry of Health, Labour and Welfare (2006) Outline for the results of the National Health and Nutrition Survey Japan.

http://www.nih.go.jp/eiken/english/research/pdf/nhns200 6_outline.pdf

[3] Ministry of Health, Labour and Welfare (2007) Chapter 4: Future health promotion and medicine-Reforming the medical architecture. In: The white paper of the Ministry of Health and Welfare in Heisei 19, KouseiToukeiKyokai, Tokyo, 97-161.

[4] Muramoto, A., Yamamoto, N., Nakamura, M., et al. (2010) Effect of intensive lifestyle intervention programs on metabolic syndrome and obesity: How much weight reduction is needed to improve metabolic comorbidities? Journal of Japan Society for the Study of Obesity, 16, 182-187.

[5] Moriguchi, J., Matsuo, F., Ejima, K., et al. (2011) Effectiveness of specific health guidance against metabolic syndrome. Official Journal of Japan Society of Ningen Dock, 26, 75-79.

[6] Ohnishi, C., Eba, I., Fukuchi, K., et al. (2011) Effect of specific health guidance on patients with extremely high serum triglyceride levels. Official Journal of Japan Society of Ningen Dock,25, 831-836.

[7] Ministry of Health and Welfare. (2010) About implementation status of specific health checkup and specific health guidance in 2008.

http://www.mhlw.go.jp/bunya/shakaihosho/iryouseido01/ pdf/info03n-01.pdf

[8] Ministry of Health and Welfare. (2011) About implementation status of specific health checkup and specific health guidance in 2009. http://www.mhlw.go.jp/bunya/shakaihosho/iryouseido01/ dl/info03 h21 00.pdf

[9] Braeckman, L., Maes, L., Bellemans, M., et al. (1998) Workers participation in a nutrition education programme. Archives of Public Health, 56, 275-289.

[10] Groeneveld, I.F., Proper, K.I., van der Beek, A.J., et al. (2009) Factors associated with non-participation and dropout in a lifestyle intervention for workers with an elevated risk of cardiovascular disease. The International Journal of Behavioral Nutrition and Physical Activity, 6, 80. doi:10.1186/1479-5868-6-80

[11] Kruger, J., Yore, M.M., Bauer, D.R., et al. (2007) Selected barriers and incentives for worksite health promo- tion services and policies. American Journal of Health Promotion, 21, 439-447. doi:10.4278/0890-1171-21.5.439

[12] Middlestadt, S.E., Sheats, J.L., Geshnizjani, A., et al. (2011) Factors associated with participation in work-site wellness programs: Implications for increasing willingness among rural service employees. Health Education and Behavior, 38, 502-509. doi:10.1177/1090198110384469

[13] Anspaugh, D.J., Hunter, S. and Savage, P. (1996) Enhancing employee participation in Corporate Health Promotion Programs. American Journal of Health Behavior, 20, 112-120.

[14] Mavis, B.E., Stachnik, T.J., Gibson, C.A., et al. (1992) Issues related to participation in worksite health promotion: A preliminary study. American Journal of Health Promotion, 7, 53-60. doi:10.4278/0890-1171-7.1.53

[15] Gucciardi, E., Cameron, J.I., Liao, C.D., et al. (2007) Program design features that can improve participation in health education interventions. BMC Medical Research Methodology, 7, 47. doi:10.1186/1471-2288-7-47

[16] Veitch, J., Clavisi, O. and Owen, N. (1999) Physical activity initiatives for male factory workers: Gatekeepers' perceptions of potential motivators and barriers. Australian and New Zealand Journal of Public Health, 23, 505-510. doi:10.1111/j.1467-842X.1999.tb01307.x

[17] Alexy, B.B. (1991) Factors associated with participation or nonparticipation in a workplace wellness center. $R e$ search in Nursing and Health, 14, 33-40.

[18] Fletcher, G.M., Behrens, T.K. and Domina, L. (2008) Barriers and enabling factors for work-site physical activity programs: a qualitative examination. Journal of Physical Activity andHealth, 5, 418-429.

[19] Sallis, J.F., Hovell, M.F. and Hofstetter, C.R. (1992) Predictors of adoption and maintenance of vigorous physical activity in men and women. Preventive Medicine, 21, 237-251. doi:10.1016/0091-7435(92)90022-A

[20] Janz, N.K. and Becker, M.H. (1984) The Health Belief Model: A decade later. Health Education Quarterly, 11, 1-47. doi:10.1177/109019818401100101

[21] Champion, V.L. and Skinner, C.S. (2008) The Health Belief Model. In: Glanz, K., Rimer, B.K. and Viswanath, K. Eds., Health behavior and health education: Theory, research, and practice. 4th Edition, Jossey-Bass, San Francisco, 189-193.

[22] Blue, C.L. and Valley, J.M. (2002) Predictors of influenza vaccine. Acceptance among healthy adult workers. Official Journal of the American Association of Occupational Health Nurses, 50, 227-233.

[23] Shahrabani, S. and Benzion, U. (2010) Workplace vaccination and other factors impacting influenza vaccination decision among employees in Israel. International Journal of Environmental Research and Public Health, 7, 853-869. doi:10.3390/ijerph7030853

[24] Hay, J.L., Ford, J.S., Klein, D., et al. (2003) Adherence to colorectal cancer screening in mammography-adherent older women. Journal of Behavioral Medicine, 26, 553-576. doi:10.1023/A:1026253802962

[25] Cam, O. and Gumus, A.B. (2009) Breast cancer screening 
behavior in Turkish women: Relationships with health beliefs and self-esteem, body perception and hopelessness. Asian Pacific Journal of Cancer Prevention, 10, 49-56.

[26] Carpenter, C.J. (2010) A meta-analysis of the effectiveness of health belief model variables in predicting behave- ior. Health Communication, 25, 661-669. doi:10.1080/10410236.2010.521906

[27] Painter, J.E., Sales, J.M., Pazol, K., et al. (2010) Psycho- social correlates of intention to receive an influenza vaccination among rural adolescents. Health Education Research, 25, 853-864. doi:10.1093/her/cyq037

[28] Morowatisharifabad, M.A. (2009) The Health Belief Model variables as predictors of risky driving behaviors among commuters in Yazd, Iran. Traffic Injury Prevention, 10, 436-440. doi:10.1080/15389580903081016 